\title{
Controlling Positronium Annihilation with Electric Fields
}

\author{
A. M. Alonso, B. S. Cooper, A. Deller, S. D. Hogan, and D. B. Cassidy \\ Department of Physics and Astronomy, University College London, Gower Street, London WClE 6BT, United Kingdom
}

(Received 2 September 2015; published 27 October 2015)

\begin{abstract}
We show that the annihilation dynamics of excited positronium (Ps) atoms can be controlled using parallel electric and magnetic fields. To achieve this, Ps atoms were optically excited to $n=2$ sublevels in fields that were adjusted to control the amount of short-lived and long-lived character of the resulting mixed states. Inclusion of the former offers a practical approach to detection via annihilation radiation, whereas the increased lifetimes due to the latter can be exploited to optimize resonance-enhanced two-photon excitation processes (e.g., $1^{3} S \rightarrow 2^{3} P \rightarrow n S / n D$ ), either by minimizing losses through intermediate state decay, or by making it possible to separate the excitation laser pulses in time. In addition, photoexcitation of mixed states with a $2^{3} S_{1}$ component represents an efficient route to producing long-lived pure $2{ }^{3} S_{1}$ atoms via single-photon excitation.
\end{abstract}

DOI: 10.1103/PhysRevLett.115.183401

PACS numbers: 36.10.Dr, 78.70.Bj

Positronium (Ps) is an atomic system composed of an electron bound to a positron and has several unique properties, the most striking of which are its low mass $\left(M_{\mathrm{Ps}}=2 m_{e}\right)$ and the fact that it can decay through various annihilation pathways [1]. Electron-positron annihilation requires overlap of the particle wave functions [2] and in Ps is therefore dependent on the square of the radial wave function at the origin. This is zero for states with $\ell>0$ [3]. Higher order annihilation processes can occur for states with $\ell>0$ but are strongly suppressed [4] and in practice direct Ps annihilation occurs only from the ground $1{ }^{1} S_{0}$ and $1^{3} S_{1}$, and metastable $2^{1} S_{0}$ and $2^{3} S_{1}$ levels. The inhibition of Ps annihilation in states with $\ell>0$ is the basis for several proposed schemes to manipulate annihilation rates using resonant and nonresonant laser fields [5-11]. More complex strategies have also been suggested [12,13], although these have also not yet been experimentally demonstrated.

The radiative decay and annihilation time scales in the $n=2$ manifold in Ps span an enormous range: The $2^{1} S_{0}$ level is radiatively metastable, fluorescing on a time scale of $\simeq 0.24 \mathrm{~s}$ [3], but annihilating in $1 \mathrm{~ns}$; conversely the $2^{3} P_{0,1,2}$ levels decay by fluorescence to the $1^{3} S_{1}$ state in $3.19 \mathrm{~ns}$, but their annihilation lifetimes exceed $100 \mu \mathrm{s}$ [4]. The $2^{3} S_{1}$ level is also metastable and fluoresces in $\simeq 0.24 \mathrm{~s}$, but has an annihilation lifetime of $\sim 1.14 \mu \mathrm{s}$ [1]. It is the disparate properties of these states that make the approach described here to manipulating decay rates particularly effective.

We report the results of experiments in which Ps annihilation rates in a weak magnetic field are manipulated using parallel electric fields. This combination of fields permits control over the spin multiplicity and orbital angular momentum character of the $n=2$ sublevels accessible by single-photon excitation from the $1^{3} S_{1}$ ground state. By increasing the $2^{1} P$ character of mixed states in the fields, radiative decay to the short-lived singlet ground state $\left({ }^{1} S_{0}\right)$ can be maximized. Similarly, mixing in $2^{1} S$ character can lead to $n=2$ annihilation. These rapid annihilation pathways can be exploited to trigger efficient and controllable detection. Conversely, introducing $2^{3} S$ character to short-lived $2^{3} P$ levels can significantly increase their lifetimes, reducing losses by radiative decay and increasing the efficiency of multiphoton excitation toward higher-lying states. Single-photon excitation of mixed states which possess $2^{3} S$ and $2^{3} P$ character will also permit isolation of samples of pure $2^{3} S_{1}$ atoms if excitation is followed by extraction from the electric field.

The experimental procedures employed here have been described elsewhere [14]. Positrons captured from a $\beta$-decay source are stored in a Surko-type Penning trap [15] from which pulses with $\sim 10^{5}$ particles are ejected each second. These pulses have Gaussian spatial distributions with full width at half maxima (FWHM) of $\sim 3 \mathrm{~mm}$ on target, and are $<5 \mathrm{~ns}$ in duration. The positrons are accelerated to $2.5 \mathrm{keV}$ and implanted into a porous silica $\left(\mathrm{SiO}_{2}\right)$ film [16] in a magnetic field of $13 \mathrm{mT}$. Ps atoms are produced with an efficiency of $\sim 30 \%$ and a mean kinetic energy of $\sim 50 \mathrm{meV}$ [17]. Following emission from the $\mathrm{SiO}_{2}$ target the Ps atoms form a rapidly expanding dilute gas with an initial density of $\sim 10^{7} \mathrm{~cm}^{-3}$. These atoms were excited via single-photon $1^{3} S_{1} \rightarrow 2^{3} P_{0,1,2}$ transitions, driven using pulsed ( $\sim 6 \mathrm{~ns}$ FWHM) ultraviolet laser radiation with a wavelength of $\simeq 243.01 \mathrm{~nm}$. A laser with a large bandwidth $(85 \mathrm{GHz})$ was employed in order to address a significant fraction of the Doppler broadened $(>500 \mathrm{GHz})$ transition linewidth [17]. Peak laser intensities of $\leq 0.5 \mathrm{MW} / \mathrm{cm}^{2}$ were obtained, corresponding to Rabi frequencies $\leq 20 \mathrm{GHz}$, and in principle permitting $1^{1} S_{0}$ [decay rate, $\Gamma_{\text {ann }}^{S}=(125 \mathrm{ps})^{-1}=8 \mathrm{GHz}$ ] and $1^{3} S_{1}$ $\left[\Gamma_{\text {ann }}^{T}=(142 \mathrm{~ns})^{-1}=7 \mathrm{MHz}\right]$ states to be excited. However, owing to the few nanosecond emission time 
from the $\mathrm{SiO}_{2}$ target [18], only atoms in the $1^{3} S_{1}$ state interacted with the laser.

The electric field in the photoexcitation region in front of the $\mathrm{SiO}_{2}$ target was controlled by a metallic grid, oriented parallel to the target, through which the incident positron beam passed. In this configuration electric fields up to $3 \mathrm{kV} / \mathrm{cm}$ could be applied without significantly affecting the properties of the ground state Ps atoms. Ps annihilation radiation was measured using single-shot lifetime spectroscopy [19]. A $\mathrm{PbWO}_{4}$ scintillator, optically coupled to a photomultiplier tube (PMT) [20], was located approximately $5 \mathrm{~cm}$ from the target region. The PMT anode was connected directly to a fast oscilloscope, such that the timedependent voltage output constituted a lifetime spectrum. The quantity of long-lived Ps atoms is characterized by a parameter $f$, the fraction of the total integrated lifetime spectrum in a selected time window, here 30-600 ns. Changes in Ps decay rates arising from laser excitation are characterized by comparing $f$ values obtained with $\left(f_{\text {on }}\right)$, and without $\left(f_{\text {off }}\right)$ the laser present, via the parameter $S_{\gamma}=\left(f_{\text {off }}-f_{\text {on }}\right) / f_{\text {off }}$. $S_{\gamma}$ therefore represents the fractional change in the number of longer-lived excited Ps atoms present relative to the unperturbed $1^{3} S_{1}$ population. Thus, positive (negative) $S_{\gamma}$ values indicate increased (decreased) annihilation rates relative to the $1^{3} S_{1}$ decay [14].

The effects of the combined Stark and Zeeman interactions on the manifold of $n=2$ states were calculated by determining the eigenvalues and eigenvectors of the corresponding Hamiltonian matrix in an $\left|n S \ell J M_{J}\right\rangle$ basis. Following the convention of Bethe and Salpeter [3], $S$ is the total spin quantum number of the electron-positron system, $\ell$ is a single-particle orbital angular momentum quantum number, $J$ is the total orbital angular momentum quantum number, and $M_{J}$ is the projection of $J$ onto the $z$ axis defined by the external fields. In this basis the diagonal matrix elements correspond to the energies of the $2^{1} S_{0}$, $2^{1} P_{1}, 2^{3} S_{1}$, and $2^{3} P_{0,1,2}$ levels in the absence of the fields $[21,22]$.

The weak magnetic field $\vec{B}=(0,0, B)$ couples states for which $\Delta \ell=0, \Delta M_{J}=0$, and $\Delta S= \pm 1$; i.e., it couples singlet and triplet levels with the same orbital angular momentum. On the other hand, the parallel electric field $\vec{F}=(0,0, F)$ couples states for which $\Delta \ell= \pm 1, \Delta M_{J}=0$, and $\Delta S=0$; i.e., it couples $2 S$ and $2 P$ levels of the same spin multiplicity. Calculating the eigenvalues of the Hamiltonian for $B=13 \mathrm{mT}$ in a range of electric fields leads to the energy level diagram in Fig. 1(a). As the electric field increases the $2 S$ and $2 P$ terms, which are nondegenerate in zero field, gradually mix, giving rise to approximately linear Stark energy shifts for $F \gtrsim 1 \mathrm{kV} / \mathrm{cm}$. Under these conditions the wave functions of the outermost sublevels, shifted to higher and lower energies in the field, contain approximately equal amounts of $2 S$ and $2 P$ character.

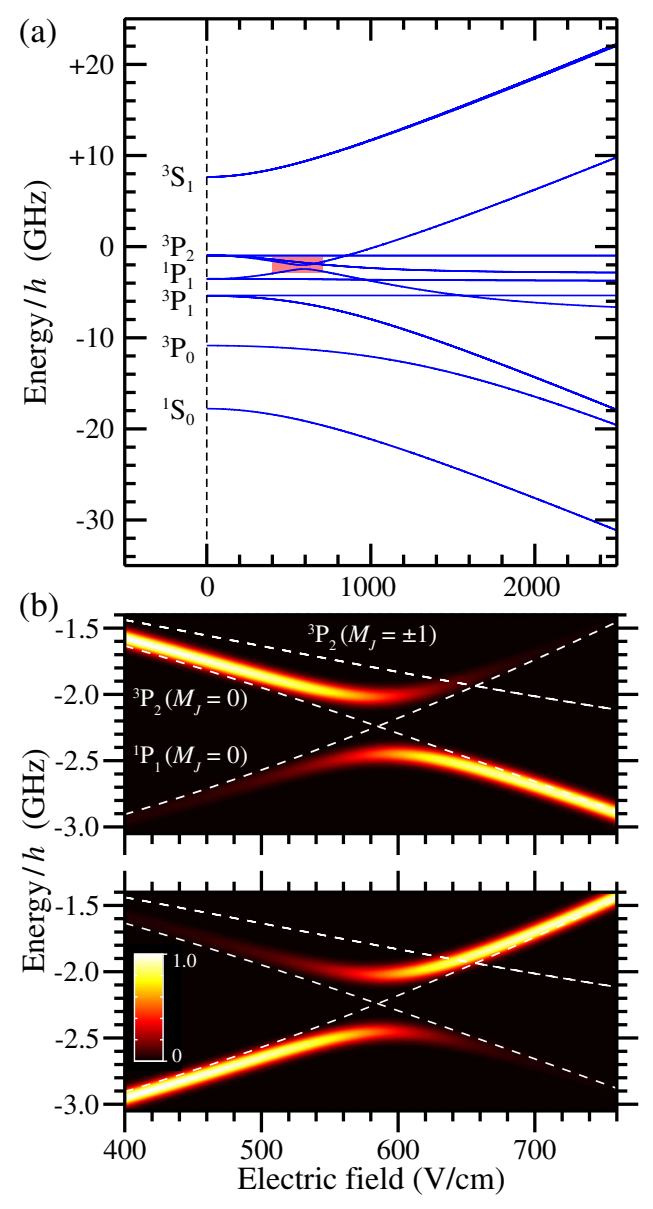

FIG. 1 (color online). (a) Calculated Stark energy level diagram for $n=2$ levels of Ps in a parallel magnetic field of $B=13 \mathrm{mT}$. The energies on the vertical axis are displayed with respect to the $n=2$ energy given by the Rydberg formula. The $85 \mathrm{GHz}$ bandwidth of the laser encompasses the entire $n=2$ Stark manifold. (b) Expanded view of the region at the avoided crossing between the $2^{3} P_{2}\left(M_{J}=0\right)$ and $2^{1} P_{1} \quad\left(M_{J}=0\right)$ sublevels highlighted by the shaded red rectangle in (a). The color map in the upper [lower] panel in (b) indicates the $2^{3} P_{2}$ $\left(M_{J}=0\right)\left[2^{1} P_{1}\left(M_{J}=0\right)\right]$ character of each sublevel at the avoided crossing. The dashed lines in each panel indicate the Stark shifts of each sublevel when $B=0$. The color scale in the lower panel is common to both.

Although the $13 \mathrm{mT}$ magnetic field only weakly perturbs the energies of the Stark sublevels, the singlet-triplet mixing induced by this field does introduce nonzero singlet character into the $\ell$-mixed triplet Stark states. This does not significantly affect the excited state decay pathways when $F=0$, but has a noticeable effect in fields close to $F=$ $585 \mathrm{~V} / \mathrm{cm}$ as can be seen in Fig. 1(b). In this figure the Stark energies of the states in zero magnetic field are indicated by the dashed curves, while those for $B=13 \mathrm{mT}$ are indicated by the color maps representing the fractional $2^{3} P_{2}\left(M_{J}=0\right)$ (upper panel), and $2^{1} P_{1}\left(M_{J}=0\right)$ (lower panel) character of each. At this position in the Stark map 
the singlet-triplet mixing induced by the magnetic field is enhanced. This is because the electric field shifts the states that adiabatically evolve to the $2^{3} P_{2}\left(M_{J}=0\right)$ and $2^{1} P_{1}$ $\left(M_{J}=0\right)$ sublevels in zero-field such that they are almost degenerate. The coupling induced by the magnetic field then gives rise to an avoided crossing [Fig. 1(b)] at which each of the interacting states possess $\sim 50 \% 2^{3} P_{2}\left(M_{J}=0\right)$ and $\sim 50 \% 2^{1} P_{1}\left(M_{J}=0\right)$ character, permitting efficient photoexcitation from the $1^{3} S_{1}$ state followed by fluorescence to the short lived $1{ }^{1} S_{0}$ state.

The effects of the fields on the annihilation dynamics of the excited Ps atoms were observed experimentally by tuning the wavelength of the laser to $243.01 \mathrm{~nm}$ and scanning the electric field in the excitation region. The results of such measurements, with the laser radiation linearly polarized parallel and perpendicular to the $z$ axis, are presented in Figs. 2(a) and 2(b), respectively. The effects of the laser polarization arise from the characteristics of the $n=2$ excited states that are accessible under the selection rules for electric-dipole transitions from the $M_{J}=0, \pm 1$ sublevels of the $1^{3} S_{1}$ state in each case. With the laser radiation polarized parallel to the $z$ axis, Fig. 2(a), $\Delta M_{J}=0$ transitions to the outer triplet Stark states dominate when $F \gtrsim 1 \mathrm{kV} / \mathrm{cm}$. Because of their mixed $2^{3} S$ and $2^{3} P$ character these excited states are longer lived than the $1^{3} S_{1}$ state, and do not mix significantly with the more rapidly annihilating singlet states. As a result $S_{\gamma}$
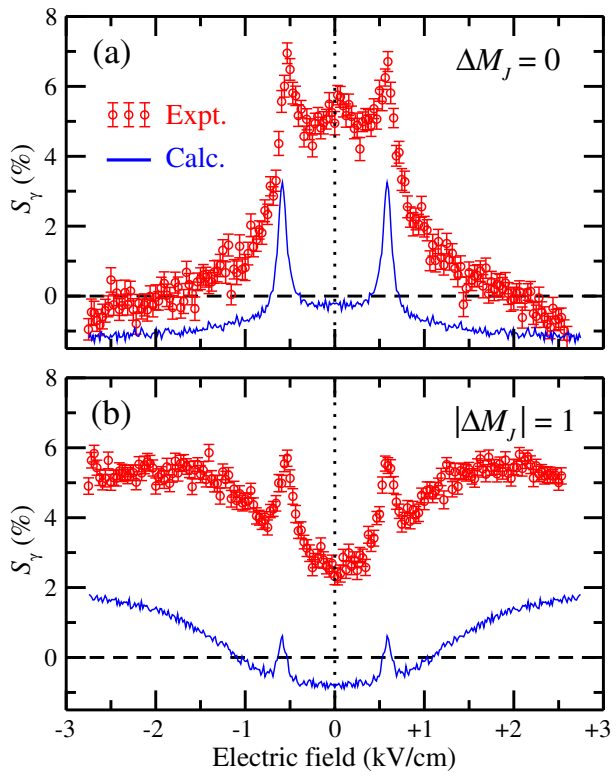

FIG. 2 (color online). Dependence of the measured Ps annihilation signal $\left(S_{\gamma}\right)$ on the electric field for photoexcitation with the laser radiation linearly polarized (a) parallel, and (b) perpendicular to the external fields $(B=13 \mathrm{mT})$. Experimental data (open circles) and the results of Monte Carlo calculations (continuous curves) are displayed. The calculated values of $S_{\gamma}$ incorporate only experimental parameters and have not been arbitrarily scaled. becomes slightly negative in the highest of these fields. When $F \lesssim 1 \mathrm{kV} / \mathrm{cm}$, the dipole allowed transitions from the $M_{J}=0$ sublevel of the ground state to the singlet-triplet mixed $2 P$ terms (see Fig. 1) permits fluorescence to the $1^{1} S_{0}$ state, followed by rapid annihilation in $\sim 125$ ps [1]. The resonant behavior of this process, which peaks at $\sim 585 \mathrm{~V} / \mathrm{cm}$, is a consequence of the significant spectral intensity of the transition to these states in this field [see Fig. 1(b)], and the approximately equal probability for decay to the singlet or triplet ground states. With the laser radiation polarized perpendicular to the $z$ axis, Fig. 2(b), $\Delta M_{J}= \pm 1$ transitions to states in the middle of the $n=2$ Stark manifold are preferentially excited when $F \gtrsim 1 \mathrm{kV} / \mathrm{cm}$. These states exhibit significant singlettriplet mixing and an increased rate of annihilation, giving rise to positive $S_{\gamma}$ values. The Stark-tuned resonance associated with the transition to the singlet-triplet mixed state at $\sim 585 \mathrm{~V} / \mathrm{cm}$ is also accessible in this laser polarization, from the $M_{J}= \pm 1$ sublevels of $1^{3} S_{1}$ state. From these data it is evident that by selecting the polarization and propagation direction of the laser radiation with respect to the external fields, significant control can be exerted over Ps annihilation dynamics.

The interpretation of the experimental data in Fig. 2 is further aided by comparison with the results of calculations of the average $n=2$ decay rates weighted by the spectral intensity of the transitions from the $1^{3} S_{1}$ state (see Fig. 3). For each electric field the coefficients of the eigenvectors of

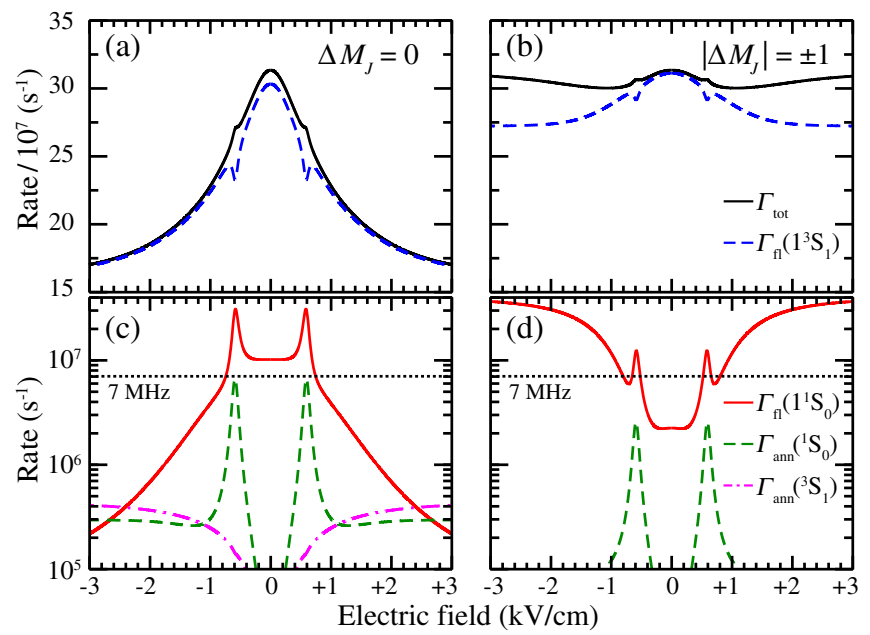

FIG. 3 (color online). Spectral-intensity-weighted average $n=$ 2 decay rates when $B=13 \mathrm{mT}$ for photoexcitation via (a) and (c) $\Delta M_{J}=0$, and (b) and (d) $\left|\Delta M_{J}\right|=1$ transitions from the $1^{3} S_{1}$ level. (a) and (b), total $n=2$ decay rates $\Gamma_{\text {tot }}$ (continuous black curve) and triplet fluorescence rate $\Gamma_{\mathrm{fl}}\left(1^{3} S_{1}\right)$ (dashed blue curve). (c) and (d), fluorescence rate to the $1^{1} S_{0}$ level, $\Gamma_{\mathrm{fl}}\left(1^{1} S_{0}\right)$, (continuous red curve), and direct $2^{1} S_{0}, \Gamma_{\text {ann }}\left(1 S_{1}\right)$, (dashed green curve) and $2^{3} S_{1}, \Gamma_{\text {ann }}\left({ }^{3} S_{1}\right)$, (dash-dotted magenta curve) annihilation rates. The horizontal dashed lines in (c) and (d) correspond to the $7 \mathrm{MHz}$ annihilation rate of the $1^{3} S_{1}$ state. 
the Stark sublevels were used to determine the transition strength to each state. These data were also employed to calculate the fluorescence and direct annihilation rates of each of the $16 n=2$ sublevels. For both laser polarizations the dominant decay pathway of the excited states is via fluorescence back to the $1^{3} S_{1}$ state [Figs. 3(a) and 3(b)]. This process does not give rise to a significant change in $S_{\gamma}$. However, the next fastest decay pathway is fluorescence from states with $2^{1} P_{1}$ character, to the $1^{1} S_{0}$ state [see Figs. 3(c) and 3(d)] followed by rapid annihilation. Comparison of the dependence of this decay process on the electric field with the experimental data in Fig. 2 confirms that it is responsible for the changes observed in the measured values of $S_{\gamma}$. In electric fields close to the avoided crossing in Fig. 1(b), local maxima are seen in the rate of fluorescence to the $1{ }^{1} S_{0}$ state. In these fields there are contributions from direct annihilation via the $2^{1} S_{0}$ level, but the rates for this process are smaller than the fluorescence rates. The average decay rates of the sublevels accessible via $\Delta M_{J}=0$ transitions from the $1^{3} S_{1}$ state decrease by a factor of $\sim 2$ when $F$ changes from zero to $3 \mathrm{kV} / \mathrm{cm}$ [Fig. 3(a)], while there is little change in the average decay rates of the states populated via $\left|\Delta M_{J}\right|=1$ transitions [Fig. 3(b)].

To associate the calculated decay rates of the excited states with the measured values of $S_{\gamma}$, a Monte Carlo calculation was performed using the spectral-intensityweighted average $n=2$ decay rates in Fig. 3. In these calculations initial samples of $2 \times 10^{5}$ atoms in the $1^{3} S_{1}$ state were generated at time zero with characteristic annihilation rates $\Gamma_{\text {ann }}^{T}=7 \mathrm{MHz}$. After a delay of $20 \mathrm{~ns}$ to account for the emission and flight of the Ps atoms to the position of the laser, half of the remaining atoms were excited via the allowed electric dipole transitions to states with $n=2$ (assuming saturation of the photoexcitation process). The decay pathways of these atoms were then tracked and the number of annihilation events in each $1 \mathrm{~ns}$ interval recorded and used to calculate $S_{\gamma}$ (continuous blue curves in Fig. 2). The common trends displayed by the experimental data and the results of the Monte Carlo calculations suggest that the physical mechanisms giving rise to the changes in the value of $S_{\gamma}$ observed in the experiments are correctly attributed to the decay processes discussed above. However, the differences between these data sets indicate that additional mechanisms beyond those included in the simple Monte Carlo model play an important role in the experiments. These arise primarily from the interplay between optical pumping, fluorescence, and annihilation in the presence of the laser field and will be the subject of future work.

The application of electric and magnetic fields to control atomic and molecular photoexcitation and decay pathways is well established (e.g., [23]) and has many important applications, such as accessing otherwise forbidden transitions for ultraprecise metrology [24], or quenching long-lived states for detection via fluorescence [25]. As demonstrated here, the Ps atom is also amenable to these methods, with the significant distinction that it can be controlled in such a way as to cause (or inhibit) annihilation. This has many useful applications including (1) significantly enhancing magnetic quenching rates for ionization-free detection, (2) the production of excited Ps atoms via multiphoton excitation schemes, and (3) singlephoton production of $2^{3} S_{1}$ states.

Increasing a magnetic quenching signal via Stark mixing is considerably more straightforward than applying the corresponding strong magnetic field [26], and therefore could improve the signal in low-magnetic-field Ps excitation experiments $[27,28]$. The alternative, photoionization, is not always desirable as it requires a high laser fluence (typically $>20 \mathrm{~mJ} / \mathrm{cm}^{2}$ ) which can damage sensitive Ps formation targets, and may require an additional laser. It is also useful to have the ability to rapidly turn the quenching on and off via an applied electric field.

The resonance-enhanced two-photon excitation process $1^{3} S \rightarrow 2^{3} P \rightarrow n S / n D$ has been used previously to produce Rydberg Ps [29-32]. Extending the lifetimes of the $n=2$ intermediate sublevels using electric and magnetic fields offers the possibility for improved excitation efficiency without the need to employ higher $n$ intermediate states [33]. Furthermore, typical pulsed laser systems used for Ps photoexcitation have durations in the range 5-10 ns. The 3.19 ns lifetime of $2^{3} P$ levels means that in a resonanceenhanced two-photon excitation process the two lasers must overlap in time. By extending the average lifetime of the intermediate state to close to $10 \mathrm{~ns}$ this is no longer required, and the lasers can be temporally separated. This will make it possible to perform spectroscopic measurements in which the first laser does not influence the second transition, e.g., via ac Stark shifts [34].

Previously demonstrated methods for the preparation of $2^{3} S_{1}$ states of Ps involve two-photon excitation of ground state atoms [35-37], or positron impact on untreated surfaces [38-41]. However, if appropriate mixed states are extracted into an electric-field-free region then pure $2^{3} S_{1}$ atoms can be obtained. This would be easier to implement than the two-photon scheme, and significantly more efficient than collisional production. The availability of $2^{3} S_{1}$ atoms will be useful for microwave spectroscopy of the $n=2$ Ps fine structure [42-44], the application of techniques to characterize Doppler effects for improved spectroscopic resolution (e.g., $[31,45]$ ), and high-resolution laser spectroscopy of transitions to more highly excited states [46]. As it is composed only of leptons, such spectroscopy of Ps could provide a measurement of the Rydberg constant that is free of hadronic influence, which would be relevant to the ongoing proton radius puzzle as observed in muonic hydrogen experiments $[47,48]$.

We are grateful to L. Liszkay for providing silica samples and to T.E. Wall for earlier work on the laser setup. This 
research was supported by UCL through its Impact Studentship Programme, and was funded in part by the Leverhulme trust (Grant No. RPG-2013-055), the ERC (Grant No. CIG 630119), and the EPSRC (Grant No. EP/ K028774/1).

[1] A. Rich, Rev. Mod. Phys. 53, 127 (1981).

[2] P. Dirac, The Principles of Quantum Mechanics (Clarendon Press, Oxford, 1930).

[3] H. Bethe and E. Salpeter, Quantum Mechanics of One- and Two-Electron Atoms (Springer, Berlin, 1957).

[4] A. I. Alekseev, Sov. Phys. JETP 7, 826 (1958).

[5] V. Pazdzerskii, Sov. Phys. J. 20, 963 (1977).

[6] F. H. M. Faisal and P. S. Ray, J. Phys. B 14, L715 (1981).

[7] M. H. Mittleman, Phys. Rev. A 33, 2840 (1986).

[8] F. Ehlotzky, Phys. Lett. A 126, 524 (1988).

[9] A. Karlson and M. H. Mittleman, J. Phys. B 29, 4609 (1996).

[10] L. B. Madsen, L. A. A. Nikolopoulos, and P. Lambropoulos, J. Phys. B 32, L425 (1999).

[11] F. M. S. Lima, M. A. Amato, O. A. C. Nunes, A. L. A. Fonseca, and E. F. da Silva, Jr, J. Phys. B 42, 055601 (2009).

[12] J. Ackermann, J. Shertzer, and P. Schmelcher, Phys. Rev. Lett. 78, 199 (1997).

[13] N. Cui, M. Macovei, K. Z. Hatsagortsyan, and C. H. Keitel, Phys. Rev. Lett. 108, 243401 (2012).

[14] B. S. Cooper, A. M. Alonso, A. Deller, T. E. Wall, and D. B. Cassidy, Rev. Sci. Instrum. 86, 103101 (2015).

[15] J. R. Danielson, D. H. E. Dubin, R. G. Greaves, and C. M. Surko, Rev. Mod. Phys. 87, 247 (2015).

[16] L. Liszkay, C. Corbel, P. Perez, P. Desgardin, M. F. Barthe, T. Ohdaira, R. Suzuki, P. Crivelli, U. Gendotti, A. Rubbia, M. Etienne, and A. Walcarius, Appl. Phys. Lett. 92, 063114 (2008).

[17] D. B. Cassidy, P. Crivelli, T. H. Hisakado, L. Liszkay, V. E. Meligne, P. Perez, H. W. K. Tom, and A. P. Mills, Jr., Phys. Rev. A 81, 012715 (2010).

[18] A. Deller, B. S. Cooper, T. E. Wall, and D. B. Cassidy, New J. Phys. 17, 043059 (2015).

[19] D. B. Cassidy, S. H. M. Deng, H. K. M. Tanaka, and A. P. Mills, Jr., Appl. Phys. Lett. 88, 194105 (2006).

[20] D. B. Cassidy and A. P. Mills, Jr., Nucl. Instrum. Methods Phys. Res., Sect. A 580, 1338 (2007).

[21] S. M. Curry, Phys. Rev. A 7, 447 (1973).

[22] C. D. Dermer and J. C. Weisheit, Phys. Rev. A 40, 5526 (1989).

[23] B. R. Johnson, J. O. Hirschfelder, and K.-H. Yang, Rev. Mod. Phys. 55, 109 (1983).

[24] A. Derevianko and H. Katori, Rev. Mod. Phys. 83, 331 (2011).
[25] C. Zimmermann, R. Kallenbach, and T. W. Hänsch, Phys. Rev. Lett. 65, 571 (1990).

[26] D. B. Cassidy, T. H. Hisakado, H. W. K. Tom, and A. P. Mills, Phys. Rev. Lett. 106, 173401 (2011).

[27] K. P. Ziock, C. D. Dermer, R. H. Howell, F. Magnotta, and K. M. Jones, J. Phys. B 23, 329 (1990).

[28] A. Deller, D. Edwards, T. Mortensen, C. A. Isaac, D. P. van der Werf, H. H. Telle, and M. Charlton, J. Phys. B 48, 175001 (2015).

[29] K. P. Ziock, R. H. Howell, F. Magnotta, R. A. Failor, and K. M. Jones, Phys. Rev. Lett. 64, 2366 (1990).

[30] D. B. Cassidy, T. H. Hisakado, H. W. K. Tom, and A. P. Mills, Phys. Rev. Lett. 108, 043401 (2012).

[31] A. C. L. Jones, T. H. Hisakado, H. J. Goldman, H. W. K. Tom, A. P. Mills, and D. B. Cassidy, Phys. Rev. A 90, 012503 (2014).

[32] T. E. Wall, A. M. Alonso, B.S. Cooper, A. Deller, S. D. Hogan, and D. B. Cassidy, Phys. Rev. Lett. 114, 173001 (2015).

[33] F. Castelli, I. Boscolo, S. Cialdi, M. G. Giammarchi, and D. Comparat, Phys. Rev. A 78, 052512 (2008).

[34] T. E. Wall, D. B. Cassidy, and S. D. Hogan, Phys. Rev. A 90, 053430 (2014).

[35] S. Chu and A. P. Mills, Phys. Rev. Lett. 48, 1333 (1982).

[36] M. S. Fee, A. P. Mills, S. Chu, E. D. Shaw, K. Danzmann, R. J. Chichester, and D. M. Zuckerman, Phys. Rev. Lett. 70, 1397 (1993).

[37] D. A. Cooke, P. Crivelli, J. Alnis, A. Antognini, B. Brown, S. Friedreich, A. Gabard, T. W. Haensch, K. Kirch, A. Rubbia, and V. Vrankovic, Hyperfine Interact. 233, 67 (2015)

[38] K. F. Canter, A. P. Mills, and S. Berko, Phys. Rev. Lett. 34, 177 (1975).

[39] D. C. Schoepf, S. Berko, K. F. Canter, and P. Sferlazzo, Phys. Rev. A 45, 1407 (1992).

[40] T. D. Steiger and R. S. Conti, Phys. Rev. A 45, 2744 (1992).

[41] D. J. Day, M. Charlton, and G. Laricchia, J. Phys. B 34, 3617 (2001).

[42] A. P. Mills, S. Berko, and K. F. Canter, Phys. Rev. Lett. 34, 1541 (1975).

[43] S. Hatamian, R. S. Conti, and A. Rich, Phys. Rev. Lett. 58, 1833 (1987).

[44] D. Hagena, R. Ley, D. Weil, G. Werth, W. Arnold, and H. Schneider, Phys. Rev. Lett. 71, 2887 (1993).

[45] G. Hagel, R. Battesti, F. Nez, L. Julien, and F. Biraben, Phys. Rev. Lett. 89, 203001 (2002).

[46] C. Schwob, L. Jozefowski, B. de Beauvoir, L. Hilico, F. Nez, L. Julien, F. Biraben, O. Acef, J.-J. Zondy, and A. Clairon, Phys. Rev. Lett. 82, 4960 (1999).

[47] R. Pohl, R. Gilman, G. A. Miller, and K. Pachucki, Annu. Rev. Nucl. Part. Sci. 63, 175 (2013).

[48] S. G. Karshenboim, Phys. Rev. A 91, 012515 (2015). 